http://dx.doi.org/10.32929/2446-8355.2019v28n3p354-366

\title{
EFICIÊNCIA DAS LARVAS DE BESOURO COPRÓFAGO NA COMPOSTAGEM DO ESTERCO DE COELHO
}

\author{
Claudete Martins da Silva Pereira ${ }^{1}$, Luiz Fernando de Sousa Antunes ${ }^{2 *}$, Milene da Silva \\ Soares $^{3}$, Adriana Maria de Aquino ${ }^{4}$, Marco Antonio de Almeida Leal ${ }^{5}$
}

\begin{abstract}
${ }^{1}$ Docente do Colégio Técnico da Universidade Federal Rural do Rio de Janeiro - CTUR, Doutora em Tecnologia e Inovação Agropecuária (UFRRJ), Agroecologia e paisagismo ambiental, Seropédica, Rio de Janeiro.

${ }^{2}$ Doutorando do Programa de Pós-Graduação em Fitotecnia da Universidade Federal Rural do Rio de Janeiro, Mestre em Agronomia (UFRRJ), Agroecologia e substratos orgânicos, Seropédica, Rio de Janeiro. *E-mail do autor correspondente: fernando.ufrrj.agro@gmail.com

${ }^{3}$ Mestranda do Programa de Pós-Graduação em Fitotecnia da Universidade Federal Rural do Rio de Janeiro, Graduação em Agronomia (UFRRJ), Agroecologia, Seropédica, Rio de Janeiro.

${ }^{4}$ Pesquisadora da Embrapa Agrobiologia, Doutora em Agronomia (UFRRJ), Agricultura orgânica, Seropédica, Rio de Janeiro.

${ }^{5}$ Pesquisador da Embrapa Agrobiologia, Doutor em Agronomia (UFRRJ), Sistemas sustentáveis de produção, Seropédica, Rio de Janeiro.
\end{abstract}

Recebido: 22/05/2019; Aceito: 10/10/2019

RESUMO: As famílias de coleópteros atuam na fragmentação da matéria orgânica em decomposição (restos vegetais, madeira podre, palha, esterco, entre outros), participando da ciclagem de nutrientes e contribuindo para a melhoria da fertilidade e estrutura do solo. $\mathrm{O}$ presente trabalho teve o objetivo de avaliar a eficiência das larvas de besouro da subfamília Cetoniinae como uma nova alternativa no processo de decomposição do esterco de coelho, comparando os atributos físicos, físico-químicos e químicos do Cetoniinaecomposto com o esterco de coelho decomposto por minhocas e o esterco compostado naturalmente. $\mathrm{O}$ experimento foi implantado em galpão coberto da Embrapa Agrobiologia, no município de Seropédica (RJ), em um delineamento experimental inteiramente casualizado com três formas de incubação: com minhocas (T1), com larvas de besouro (T2) e sem larvas e sem minhocas (T3), com quatro repetições. As coletas de amostras do material para análise foram realizadas no início da incubação (tempo 0) e aos 30, 60 e 90 dias, sendo avaliados pH e emissões potenciais de $\mathrm{CO}_{2}$ e de $\mathrm{NH}_{3}$ como indicadores de estabilidade, e aos 90 dias foram analisadas as seguintes variáveis: condutividade elétrica $(\mathrm{CE}), \mathrm{pH}$, teores de nitrogênio, fósforo, potássio, cálcio e magnésio, e substâncias húmicas. Os compostos de esterco de coelho produzidos por larvas de Cetoniinae (Cetoniinaecomposto), por minhocas (vermicomposto) e esterco compostado, não apresentaram diferença significativa para a maioria das características químicas e físicas, constatando-se a estabilização dos compostos produzidos por minhocas e larvas aos 90 dias, medida pelas emissões de $\mathrm{CO}_{2}$ e de $\mathrm{NH}_{3}$. Destaca-se a decomposição realizada pelas larvas de besouro, a qual apresentou a menor emissão de $\mathrm{NH}_{3}$ ao longo do período de incubação, demonstrando que as larvas podem ser uma nova alternativa a compostagem do esterco de coelho.

Palavras-chave: Cetoniinae. Resíduos orgânicos. Compostagem. Nutrientes. 


\title{
EFFICIENCY OF COPROPHAGE BEETLE LARVAE ON COMPOSTING OF RABBIT MANURE
}

\begin{abstract}
Coleoptera families act in the fragmentation of decaying organic matter (vegetable debris, rotten wood, straw, manure, among others), participating in nutrient cycling and contributing to improved soil fertility and structure The present work aimed to evaluate the efficiency of Cetoniinae subfamily beetle larvae as a new alternative in the decomposition process of rabbit manure, comparing the physical, physicochemical and chemical attributes of Cetoniinacompost with the earthworm decomposed rabbit manure and the naturally composted manure. The experiment was carried out in a covered warehouse of Embrapa Agrobiologia, in the city of Seropédica (RJ), in a completely randomized experimental design with three incubation forms: with earthworms (T1), with beetle larvae (T2) and without larvae and without earthworms (T3), with four repetitions for each treatment. Samples of material for analysis were collected at the beginning of incubation (time 0) and at 30, 60 and 90 days, being that the $\mathrm{pH}$ and potential emissions of $\mathrm{CO}_{2}$ and $\mathrm{NH}_{3}$ were evaluated as indicators of stability, and at 90 days the following variables were analyzed: electrical conductivity (EC), $\mathrm{pH}$, nitrogen, phosphorus, potassium, calcium and magnesium contents, and humic substances. Rabbit manure compounds produced by Cetoniinae (Cetoniinaecompost) larvae, worms (vermicompost) and composted manure showed no significant difference for most of the chemical and physical characteristics, measured by the emissions of $\mathrm{CO}_{2}$ and $\mathrm{NH}_{3}$. We highlight that decomposition performed by the beetle larvae, which presented the lowest $\mathrm{NH}_{3}$ emission during the incubation period, demonstrating that larvae may be a new alternative to rabbit manure composting.
\end{abstract}

Keywords: Cetoniinae. Organic wastes. Composting. Nutrients.

\section{INTRODUÇÃO}

A agricultura orgânica representa um mercado em franca expansão (LUIZZI et al., 2016), e tem como princípio manter e/ou aumentar a fertilidade do solo ao longo do tempo, especialmente a partir de recursos renováveis e locais (BRASIL, 2003). Nesse sentido, priorizam-se substratos e fertilizantes orgânicos obtidos a partir de materiais disponíveis e que sejam também de fácil aquisição.

A cunicultura atende a essas premissas, pois além possibilitar várias oportunidades de comercialização, também permite o uso do esterco na agricultura, uma vez que a relação $\mathrm{C} / \mathrm{N}$ próxima do valor indicado como referência $(\mathrm{C} / \mathrm{N} \leq 20)$, conforme Queiroz et al. (2014), o classifica como um fertilizante orgânico simples de acordo com o decreto 4954/2004

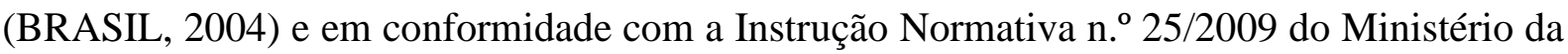
Agricultura (BRASIL, 2009). Porém, a utilização segura do esterco de coelho como fertilizante orgânico ou na formulação de substratos requer a sua prévia estabilização, sendo necessário buscar alternativas que favoreçam esse processo do ponto de vista de qualidade e rapidez. 
As minhocas processam o esterco de coelho (MOLINA et al., 2013) e o vermicomposto produzido tem apresentado bons resultados na produção agrícola (CABANILLAS et al., 2013). Gómez-Brandón et al. (2013) relataram a capacidade das minhocas em processarem esterco de coelho a partir de contínua oferta desse insumo puro e confirmaram, com base no perfil de fosfolipídios e nas medidas da atividade microbiana, alto grau de estabilização do produto final, cujo aspecto é fundamental no manejo orgânico do solo. Na natureza, além das minhocas, os coleópteros também atuam na decomposição da matéria orgânica (restos vegetais, madeira podre, húmus, palha etc.), participando da ciclagem de nutrientes, contribuindo desta maneira para a fertilidade e estrutura do solo (CROSSLEY et al., 1992).

Larvas de besouros coprófagos da subfamília Cetoniinae (Coleoptera: Scarabaeidae) foram sistematicamente encontradas em esterco de coelho na cunicultura da UFRuralRJ por Pereira (2017), o que despertou a atenção ao seu papel ecológico. Contudo, pouco se sabe sobre a ecologia dessa subfamília, provavelmente em decorrência da baixa diversidade desse grupo e inexistência de características de bioindicação (PUKER et al., 2014). No entanto, a capacidade dessas larvas em transformar as bolotas do esterco de coelho em um material uniforme despertou a atenção sobre o potencial de uso desse produto na agricultura, o que poderia abrir nossas possibilidades no processamento desse esterco.

Nesse sentido, o presente trabalho teve o objetivo de avaliar a eficiência das larvas de besouro da subfamília Cetoniinae como uma nova alternativa no processo de decomposição do esterco de coelho, avaliando-se, ao longo do processo, indicadores de estabilidade, como $\mathrm{pH}$ e emissões potenciais de $\mathrm{CO}_{2}$ e de $\mathrm{NH}_{3}$, e comparando, ao final do processo, os atributos físicos, físico-químicos e químicos do Cetoniinaecomposto com o esterco de coelho decomposto por minhocas e o esterco compostado naturalmente.

\section{MATERIAL E MÉTODOS}

O experimento foi implantado em galpão coberto da Embrapa Agrobiologia, adotandose o delineamento experimental inteiramente casualizado com três tratamentos e quatro repetições, sendo avaliadas três formas de incubação do esterco de coelho: com minhocas (T1), com larvas de besouro (T2) e sem larvas e sem minhocas (T3). A altitude do local é de 33,0 m e o clima é classificado como Aw, com chuvas concentradas no período de novembro a março, com precipitação anual média de $1213 \mathrm{~mm}$ e temperatura média anual de $24,5{ }^{\circ} \mathrm{C}$ (ANTUNES, 2017).

Cada parcela foi constituída por um recipiente plástico, tipo floreira, com volume de 5 $\mathrm{dm}^{-3}$. O esterco de coelho foi obtido no setor de Cunicultura da UFRRJ e submetido a um processo de pré-estabilização durante 30 dias, que consistiu em irrigações constantes para reduzir os efeitos nocivos de elevada salinidade e de elevadas temperaturas, tornando o material adequado para a colonização dos organismos decompositores.

$\mathrm{Na}$ etapa de estabilização foram liberadas em cada recipiente 20 minhocas adultas da espécie Eisenia andrei, coletadas no minhocário do Sistema Integrado de Produção Agroecológica (SIPA - "Fazendinha Agroecológica Km 47”), localizado em Seropédica, RJ ou 20 larvas de Scarabaeidae, subfamília Cetoniinae, sendo 10 larvas em estádio L2 e 10 em 
estádio L3, coletadas na Cunicultura da UFRRJ. Os recipientes foram cobertos com tela do tipo mosquiteiro evitando a entrada de outros organismos e oviposição de moscas.

Foram efetuadas irrigações com água destilada durante o experimento, buscando-se, manter a umidade. Aos 0, 30, 60 e 90 dias após a incubação foram coletadas amostras dos compostos orgânicos para avaliação da sua estabilidade por meio de análises de $\mathrm{pH}$ e das emissões de $\mathrm{CO}_{2}$ e $\mathrm{NH}_{3}$.

Aos 90 dias também foram avaliados a variação da massa e do volume em relação à massa e volume iniciais e variação do conteúdo de $\mathrm{N}$ em relação ao conteúdo inicial e teor final de $\mathrm{N}$, bem como condutividade elétrica, densidade, teores totais de $\mathrm{C}, \mathrm{N}, \mathrm{Ca}, \mathrm{Mg}, \mathrm{P}$ e $\mathrm{K}$, relação $\mathrm{C}: \mathrm{N}$, e quantificadas as proporções de ácido húmico (AH), ácido fúlvico (AF) e de substância húmicas $(\mathrm{AF}+\mathrm{AH})$, relação ac. húmico / ac. Fúlvico e índice de humificação.

As amostras foram acondicionadas em potes plásticos de $250 \mathrm{~mL}$, as quais foram vedadas com filme plástico PVC e armazenados em freezer $\left(-18^{\circ} \mathrm{C}\right)$ até o envio para análises no laboratório. No momento do processamento, as amostras foram retiradas dos potes plásticos e imediatamente divididas em duas sub-amostras. A primeira foi acondicionada em saco plástico e armazenada em freezer, para análise de $\mathrm{pH}, \mathrm{CE}$ e emissões de $\mathrm{CO}_{2}$ e $\mathrm{NH}_{3}$. Enquanto a outra destinou-se a análise dos teores totais de nutrientes e foi acondicionada em saco de papel, seca em estufa $\left(>72 \mathrm{~h}, 65^{\circ} \mathrm{C}\right)$ e posteriormente moída em moinho tipo Willey.

$\mathrm{O}$ pH e a CE foram avaliados em solução de água destilada (5:1 v/v), de acordo com a metodologia do Ministério da Agricultura, Pecuária e Abastecimento - MAPA (BRASIL, 2007). A densidade foi calculada utilizando uma proveta de $500 \mathrm{~mL}$, conforme método descrito pelo MAPA (BRASIL, 2008). As emissões de $\mathrm{CO}_{2}$ e de $\mathrm{NH}_{3}$ foram quantificadas conforme metodologia descrita por Oliveira et al. (2014) modificada, alterando-se a temperatura de incubação para $30^{\circ} \mathrm{C}$, ao invés dos $25^{\circ} \mathrm{C}$ do método original.

Os teores totais de $\mathrm{N}, \mathrm{Ca}, \mathrm{Mg}, \mathrm{K}$ e $\mathrm{P}$ foram avaliados por meio de digestão da amostra, conforme o método descrito por Silva (2009).

As frações químicas das substâncias húmicas foram obtidas pelo fracionamento químico da matéria orgânica do solo (MOS) com base no método Kononova-Belchikova ( $\mathrm{NaOH}$ 0,01 mol L ${ }^{-1}+\mathrm{Na}_{4} \mathrm{P}_{2} \mathrm{O}_{7}$ 0,01 mol L ${ }^{-1}$ ), descrito por Kononova (1982), com três repetições por amostra composta coletada em cada tratamento.

A análise estatística dos resultados de $\mathrm{pH}$ e emissões de $\mathrm{CO}_{2}$ e $\mathrm{NH}_{3}$ observados ao longo dos 90 dias foi realizada por meio da análise de variância do esquema parcela subdividida, com tratamento na parcela e época de amostragem na sub-parcela, em delineamento inteiramente casualizado com quatro repetições. Foram apresentados os níveis de significância de cada fator e da interação entre os fatores. O comportamento de cada tratamento ao longo do processo de compostagem foi apresentado por meio de gráficos contendo os valores médios e o erro padrão. Os resultados das características avaliadas somente aos 90 dias foram submetidos ao teste de normalidade, procedendo-se a transformação de $\log (\mathrm{x})$ dos dados não normais. Em seguida, foi realizada a análise de variância e a comparação das médias realizada por meio do teste de Snott-Knott $(p \leq 0,05)$, através do SISVAR (FERREIRA, 2014). 


\section{RESULTADOS E DISCUSSÃO}

Os resultados dos valores de $\mathrm{pH}$ apresentaram efeitos significativos do tempo de compostagem, tratamentos e da interação tempo $\times$ tratamento (Tabela 1). Quanto à emissão de $\mathrm{CO}_{2}$ ocorreram efeitos significativos para apenas para os tratamentos e tempos de compostagem. Já para a emissão de $\mathrm{NH}_{3}$ os efeitos foram significativos nos diferentes tratamentos, tempos de compostagem e interação tempo $\times$ tratamento.

Tabela 1. Análise de variância do esquema parcela dividida, com tratamento na parcela e tempo de incubação na subparcela, com os níveis de significância de cada fator e da interação entre os fatores, e os coeficientes de variação das parcelas e das subparcelas. Analysis of variance of the split plot scheme, with treatment in the plot and incubation time in the subplot, with the significance levels of each factor and the interaction between the factors, and the coefficients of variation of plots and subplots.

\begin{tabular}{cccccc}
\hline \multirow{2}{*}{ Tratamentos } & & \multicolumn{2}{c}{ Nível de significância } & \multicolumn{2}{c}{ CV\% } \\
\cline { 3 - 6 } & & Tempo (Te) & Interação Tr x Te & Parcela & Subparcela \\
\hline $\mathrm{pH}$ & $<0,001 * *$ & $<0,001 * *$ & $0,010 *$ & 1,76 & 1,36 \\
Emissão de $\mathrm{CO}_{2}$ & $0,002 * *$ & $<0,001 * *$ & $0,081 \mathrm{~ns}$ & 10,32 & 8,69 \\
Emissão de $\mathrm{NH}_{3}$ & $<0,001 * *$ & $<0,001 * *$ & $<0,001 * *$ & 47,22 & 45,25 \\
\hline
\end{tabular}

Nota: **: significativo ao nível de 1,0\%; *: significativo ao nível de 5,0\%; ns: não significativo. **: significant at the 1.0\% level; *: significant at the $5.0 \%$ level; ns: not significant.

A diferenciação entre os valores de $\mathrm{pH}$ dos tratamentos começou a ocorrer a partir dos 30 dias, quando o tratamento com minhocas (T1) apresentou uma queda constante até o final do experimento, atingindo aos 90 dias, $\mathrm{pH}$ final mais próximo à neutralidade do que os tratamentos com larvas e controle (T2 e T3), porém o controle apresentou pH mais elevado que os demais tratamentos (Figura 1).

$\mathrm{O} \mathrm{pH}$ fornece informações sobre o estado de decomposição da matéria orgânica (MO) que foi submetida a um processo de fermentação, no qual a matéria-prima crua terá reação ácida; quando neutra ou quase neutra, o composto estará estabilizado; e reação alcalina quando o composto estiver humificado (KIEHL,1985). Os valores de pH estão diretamente relacionados com a matéria-prima utilizada na vermicompostagem. Morselli et al. (1996) ao utilizarem diferentes materiais orgânicos na vermicompostagem, obtiveram valores de $\mathrm{pH}$ de 6,6 quando a matéria-prima foi a borra de café e 8,1 quando a material foi a erva-mate. Nesse trabalho, os efeitos observados são decorrentes da decomposição em função da presença ou não dos dois tipos de organismos da macrofauna do solo empregados: as larvas de Cetoniinae e as minhocas. Tal fato corrobora com os resultados encontrados por Ramanathan e Alagesan (2012), os quais ao avaliarem o processo de decomposição de resíduos de flores na Índia através de minhocas, diplópodes e sem a presença destes organismos, verificaram que a presença de diplópodes seguida pelo tratamento com presença de minhocas, foi muito mais efetiva na decomposição dos resíduos quando comparado ao processo de decomposição sem presença destes animais. Observa-se que o esterco de coelho incubado com minhoca e com larva mostrou resultados de $\mathrm{pH}$ mais próximos da neutralidade, o que pode ser atribuído a ação desses organismos, proporcionando maior aeração no esterco, influenciando na diminuição do pH. Já o esterco incubado sem a presença de larvas e minhocas, provavelmente 
por ser menos aerado, apresentou valores de $\mathrm{pH}$ mais elevados, o que corrobora Bidone (2001), ao relatar que a compostagem aeróbia provoca elevação do pH. Haimi e Huhta (1987), relatam que os valores muito elevados de $\mathrm{pH}$ podem representar perdas de nitrogênio amoniacal por volatilização.

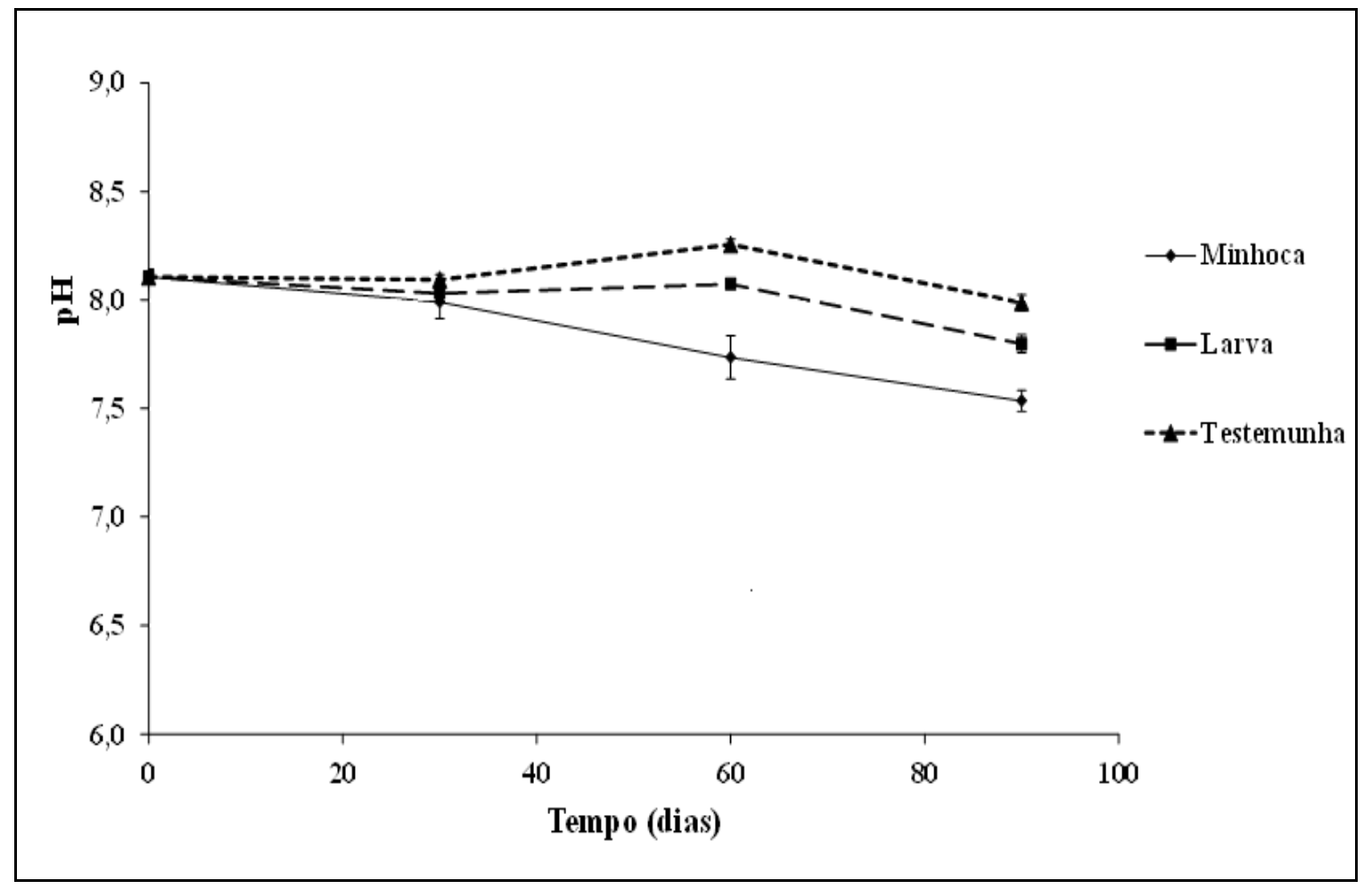

Figura 1. Valores de $\mathrm{pH}$ observados durante a incubação de esterco de coelho realizada por meio de diferentes agentes decompositores (média de quatro repetições + erro padrão). $P H$ values observed during incubation of rabbit manure by means of different decomposing agents (average of four replicates + standard error).

Conforme as curvas da emissão de $\mathrm{CO}_{2}$ apresentadas na Figura 2, no início do processo ocorreu elevada emissão de $\mathrm{CO}_{2} \mathrm{em}$ todos os tratamentos, reduzindo-se gradativamente até os 60 dias, se estabilizando aos 90 dias, no final do processo de incubação, o que indica grande atuação de organismos decompositores na fase inicial. De acordo com Oliveira et al. (2014), a emissão de $\mathrm{CO}_{2}$ está relacionada, principalmente, com o auto aquecimento e consumo de $\mathrm{O}_{2}$ pelos microrganismos.

Para Test Methods for the Examination of Composting and Compost - TMECC (2002), Bernal et al. (2009) e Wichuk e McCartney (2013), para valores de emissão de $\mathrm{CO}_{2}$ superiores a 4,0 $\mathrm{mg} \mathrm{g}^{-1} \mathrm{dia}^{-1}$, os materiais são considerados instáveis. De acordo com esta afirmação, no processo de incubação do esterco, o controle apresenta emissão de $\mathrm{CO}_{2}$ um pouco acima de 4,0 $\mathrm{mg} \mathrm{g}^{-1} \mathrm{dia}^{-1}$ aos 90 dias, enquanto os tratamentos com larva e minhocas alcançam valores menores, possivelmente pela contribuição de fragmentação, que aumenta a superfície de contato das partículas favorecendo a colonização de bactérias, fungos e atividade microbiana, influenciando na maturação do composto ao longo do processo. 


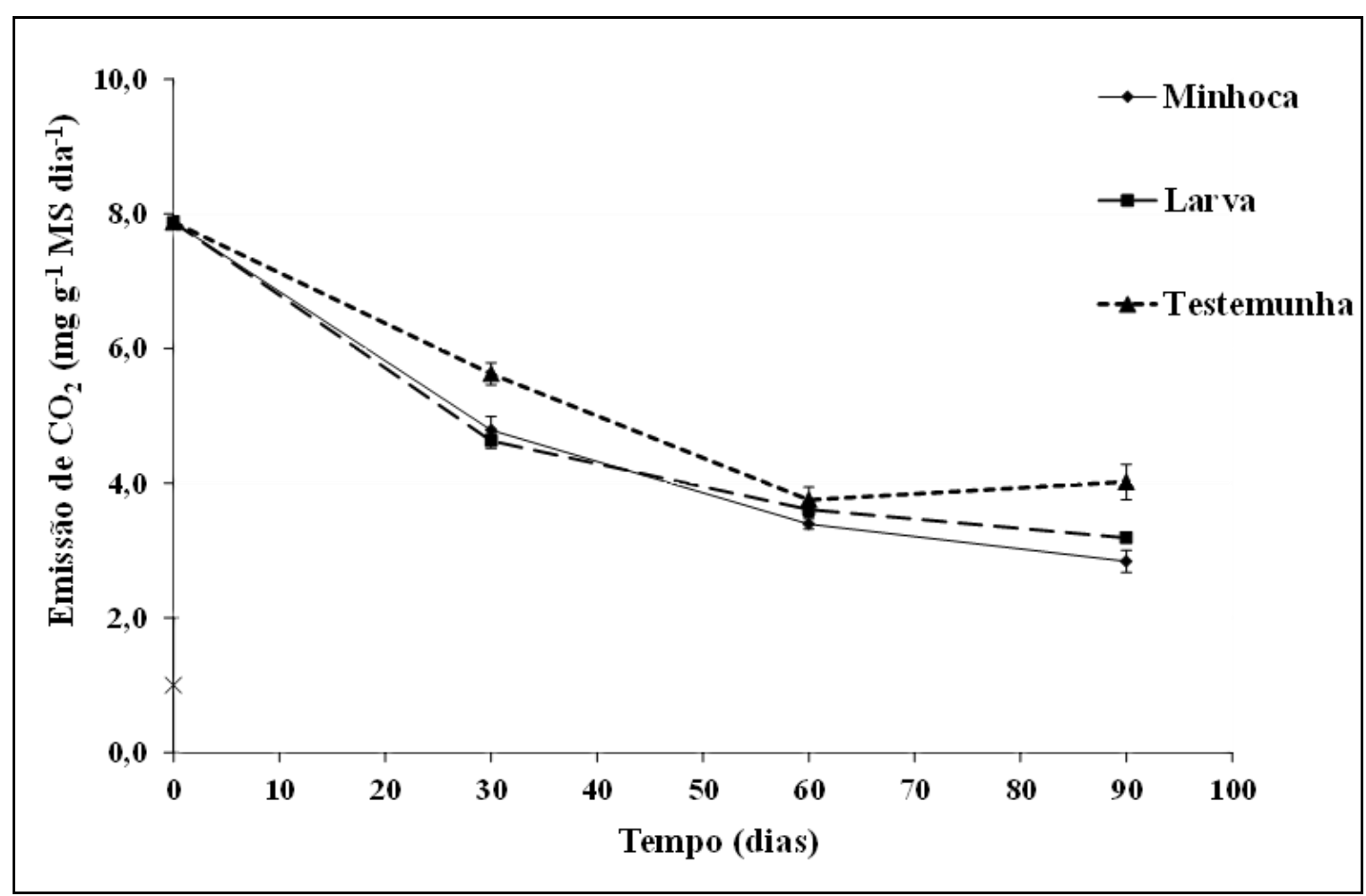

Figura 2. Emissões de $\mathrm{CO}_{2}$ observadas durante a incubação de esterco de coelho realizada por meio de diferentes processos. $\mathrm{CO}_{2}$ emissions observed during incubation of rabbit manure by different processes.

$\mathrm{O}$ aumento da emissão de $\mathrm{CO}_{2}$ do controle no final da incubação pode estar relacionado com alguma limitação dos microrganismos, pois quanto mais fina a granulometria do material compostado, maior a ação dos microrganismos (BRITO, 2005), nesse caso, a ação fragmentadora ocorrida nos tratamentos com minhocas e larvas podem favorecer uma maior interação com microrganismos do que o tratamento testemunha. De acordo com Vallini (1995) a extensão da área superficial e o grau de decomposição aumentam com a redução da dimensão das partículas.

Sabe-se que a atividade dos microrganismos decompositores nos processos de compostagem está estritamente relacionada à diversificação e a concentração de nutrientes, visto que, de acordo com Kiehl (1998), a respiração da biota é um importante indicador do grau de maturação de material orgânico. A atividade (respiração aeróbia de microrganismos quimiorganotróficos) é mais intensa, com maior liberação de $\mathrm{CO}_{2}$. No entanto, ao observarmos a Tabela 2 o final da incubação, verifica-se que os dados indicaram predominância de ácidos húmicos sobre os ácidos fúlvicos em todos os tratamentos, sendo um indicativo de humificação adequada dos resíduos avaliados. Resultados semelhantes foram obtidos por Silva (2009) ao avaliar o grau de humificação em resíduos orgânicos de origens e composição distintas no decorrer do processo de compostagem.

Os resultados de emissão de $\mathrm{NH}_{3}$ estão apresentados na Figura 3, onde observa-se que ocorreu um aumento significativo aos 30 dias nos tratamentos testemunha e com minhocas, e uma redução gradativa no tratamento com larvas. Essa perda de $\mathrm{NH}_{3}$ por volatilização, provavelmente, pode ter ocorrido devido a atuação de microrganismos que inicialmente na compostagem, metabolizaram o nitrogênio orgânico transformando-o em nitrogênio 
amoniacal, mas também Sommer e Moller (2000) e Raviv et al., (2004) que relatam que as perdas de $\mathrm{N}$ durante o processo de compostagem aumentam com a temperatura e a intensidade com que se realizam as trocas gasosas com o exterior, com a diminuição da relação $\mathrm{C} / \mathrm{N}$ e com o aumento do pH. Os processos de volatilização da amônia e de desnitrificação são responsáveis pelas maiores perdas de $\mathrm{N}$ durante a compostagem.

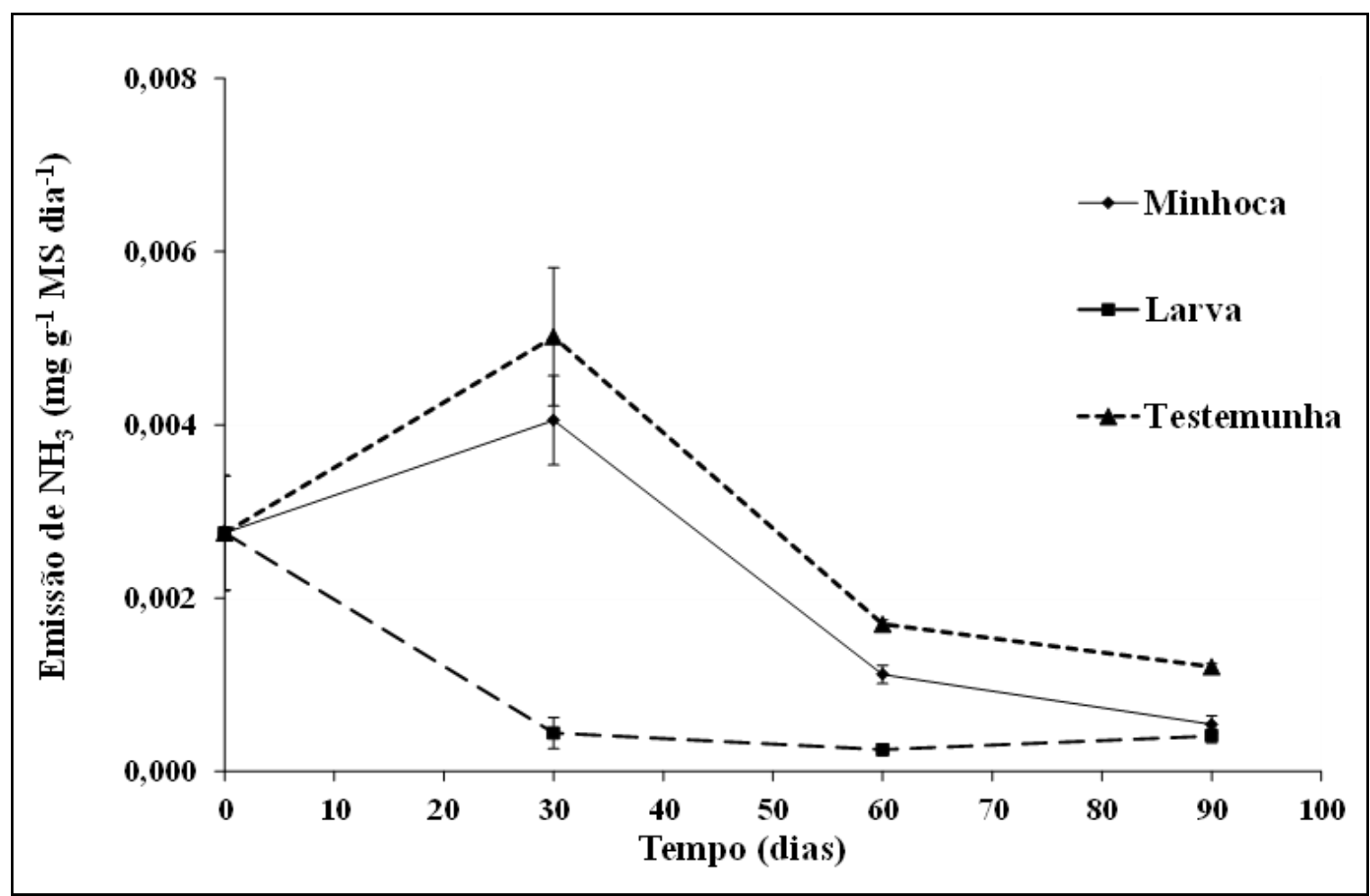

Figura 3. Emissões de $\mathrm{NH}_{3}$ observadas durante a incubação de esterco de coelho realizada por meio de diferentes processos de incubação de incubação (média de quatro repetições + erro padrão). $\mathrm{NH}_{3}$ emissions observed during incubation of rabbit manure by different incubation incubation procedures (mean of four replicates + standard error).

No decorrer da decomposição, a amônia $\left(\mathrm{NH}_{3}\right)$ pode ser perdida por volatilização ou convertida à forma de nitratos, pela nitrificação, fenômeno que é acidificante e contribui para que o composto maturado seja mais ácido do que o material original. Oliveira et al. (2014) afirmam que elevadas perdas de $\mathrm{N}$ ocorrem na compostagem de estercos devido à elevada concentração de $\mathrm{NH}_{3}$ e à presença de compostos facilmente degradáveis, que devido a baixa relação $\mathrm{C}: \mathrm{N}$, tornam existentes um excesso do $\mathrm{N}$ por $\mathrm{C}$ degradável, permitindo assim que o $\mathrm{N}$ inorgânico seja produzido em excesso, o qual pode ser perdido por volatilização de amônia ou por lixiviação do material presente no composto. Segundo Hafner et al. (2013), a emissão de $\mathrm{NH}_{3}$ a partir de dejetos animais resulta em perdas significativas de $\mathrm{N}$ nos sistemas agrícolas e pode contribuir para a poluição do ar e a degradação dos ecossistemas.

Em relação aos valores da condutividade elétrica, observa-se na Tabela 2 que o tratamento testemunha apresenta valor significativamente inferior aos demais tratamentos. A condutividade elétrica é um indicativo da concentração de sais na solução e fornece uma estimativa de salinidade nos substratos. A observação da condutividade elétrica é um importante fator, pois, o excesso de sais pode prejudicar o crescimento das plantas (GRAZIANO et al., 1995; HANDRECK; BLACK, 1999). 
Tabela 2. Caracterização físico-química, física e química dos compostos orgânicos produzidos após 90 dias de incubação do esterco de coelho contendo larvas de besouro, minhocas e testemunha (média de quatro repetições + erro padrão). Physicochemical, physical and chemical characterization of organic compounds produced after 90 days of incubation of rabbit manure containing beetle larvae, earthworms and control (mean of four replicates + standard error).

\begin{tabular}{|c|c|c|c|}
\hline \multirow{2}{*}{ Características } & \multicolumn{3}{|c|}{ Compostos } \\
\hline & Minhoca & Larva & Testemunha \\
\hline $\mathrm{pH}$ & $7,5 \mathrm{c}$ & $7,8 \mathrm{~b}$ & $8,0 \mathrm{a}$ \\
\hline Condutividade Elétrica $\left(\mu \mathrm{S} \mathrm{cm}^{-1}\right)$ & $2012 \mathrm{a}$ & $2186 \mathrm{a}$ & $1925 \mathrm{~b}$ \\
\hline Densidade $\left(\mathrm{kg} \mathrm{m}^{-3}\right)$ & $322 \mathrm{a}$ & $354 \mathrm{a}$ & $300 \mathrm{a}$ \\
\hline Teor total de $\mathrm{C}\left(\mathrm{g} \mathrm{kg}^{-1}\right)$ & 226,2 & 265,5 & 218,4 \\
\hline Relação C:N & 16,0 & 16,0 & 12,9 \\
\hline Teor total de $\mathrm{C}\left(\mathrm{g} \mathrm{kg}^{-1}\right)$ & 226,2 & 265,5 & 218,4 \\
\hline Teor total de $\mathrm{N}\left(\mathrm{g} \mathrm{kg}^{-1}\right)$ & $14,2 \mathrm{a}$ & $16,7 \mathrm{a}$ & $17,0 \mathrm{a}$ \\
\hline Teor total de $\mathrm{P}\left(\mathrm{g} \mathrm{kg}^{-1}\right)$ & $17,1 \mathrm{a}$ & $16,9 \mathrm{a}$ & $19,4 \mathrm{a}$ \\
\hline Teor total de $\mathrm{K}\left(\mathrm{g} \mathrm{kg}^{-1}\right)$ & $12,5 \mathrm{a}$ & $12,8 \mathrm{a}$ & $14,0 \mathrm{a}$ \\
\hline Teor total de $\mathrm{Ca}\left(\mathrm{g} \mathrm{kg}^{-1}\right)$ & $21,0 \mathrm{a}$ & $21,7 \mathrm{a}$ & $22,6 \mathrm{a}$ \\
\hline Teor total de $\mathrm{Mg}\left(\mathrm{g} \mathrm{kg}^{-1}\right)$ & $7,2 \mathrm{a}$ & $6,9 \mathrm{a}$ & $8,4 \mathrm{a}$ \\
\hline Proporção $(\%)$ da massa inicial $(\mathrm{CV}=13,66 \%)$ & $77,2 \mathrm{a}$ & $74,4 \mathrm{a}$ & $82,7 \mathrm{a}$ \\
\hline Proporção $(\%)$ do volume inicial $(\mathrm{CV}=6,81 \%)$ & $52,8 \mathrm{~b}$ & $43,0 \mathrm{c}$ & $63,0 \mathrm{a}$ \\
\hline Proporção $(\%)$ do N inicial $(\mathrm{CV}=17,37 \%)$ & $61,2 \mathrm{a}$ & $72,2 \mathrm{a}$ & $80,8 \mathrm{a}$ \\
\hline Ac. Húmico + Ac. Fúlvico $\left(\mathrm{g} \mathrm{kg}^{-1}, \mathrm{CV}=12,68 \%\right)$ & $2,8 \mathrm{~b}$ & $3,4 \mathrm{~b}$ & $3,9 \mathrm{a}$ \\
\hline Ac. Húmico $\left(\mathrm{g} \mathrm{kg}^{-1}, \mathrm{CV}=15,63 \%\right)$ & $1,9 \mathrm{a}$ & $2,0 \mathrm{a}$ & $2,5 \mathrm{a}$ \\
\hline Ac. Fúlvico $\left(\mathrm{g} \mathrm{kg}^{-1}, \mathrm{CV}=34,50 \%\right)$ & $0,9 \mathrm{a}$ & $1,3 \mathrm{a}$ & $1,5 \mathrm{a}$ \\
\hline Relação Ac. Húmico / Ac. Fúlvico $(\mathrm{CV}=34,50$ \%) & $2,8 \mathrm{a}$ & $1,7 \mathrm{a}$ & $1,9 \mathrm{a}$ \\
\hline Índice de Humificação $(\mathrm{CV}=15,34 \%)$ & $0,9 \mathrm{~b}$ & $0,8 \mathrm{~b}$ & $1,1 \mathrm{a}$ \\
\hline
\end{tabular}

Médias seguidas da mesma letra na linha não diferem entre si pelo teste de Scott-Knott (p<0,05); Índice de humificação $=($ ac. húmico $/ \mathrm{C}$ org $) * 100$. Averages followed by the same letter in the row do not differ from each other by the Scott-Knott test $(p<0.05)$; Humification index $=($ humic acid $/ C$ org $) * 100$.

Valores de CE entre 2000 a $4000 \mu \mathrm{S} \mathrm{cm}^{-1}$ são considerados altos para substratos. Valores de 1000 a $2000 \mu \mathrm{S} \mathrm{cm} \mathrm{cm}^{-1}$ são normais e menores que $1000 \mu \mathrm{S} \mathrm{cm} \mathrm{cm}^{-1}$ são considerados baixos (ARAÚJO NETO et al., 2009). Ao final do processo observou-se que os tratamentos com minhoca e testemunha apresentavam valores similares aos recomendados como referência e o tratamento com larvas, próximos aos valores de referência.

De acordo com Anjos et al. (2015) a concentração de nutrientes em resíduos orgânicos tende a aumentar durante o processo de vermicompostagem. Esse aumento é mais significativo para fósforo $(\mathrm{P})$, cálcio $(\mathrm{Ca})$, Magnésio $(\mathrm{Mg})$, Potássio $(\mathrm{K})$ e micronutrientes, e é atribuído a um efeito de concentração causado pela mineralização da matéria orgânica.

Observa-se na Tabela 2 que aos 90 dias de incubação não houve diferenças significativas dos teores de ac. fúlvico $(\mathrm{AF})$ e de ac. húmico $(\mathrm{AH})$, e também da relação AH/AF entre os tratamentos. Já, a testemunha apresentou teores de AF+AH e índice de humificação significativamente superior aos demais tratamentos, indicando que a incubação 
realizada sem as minhocas e sem as larvas de Cetoniinae proporcionou maior humificação que os demais tratamentos. A formação das substâncias húmicas se dá por inúmeros mecanismos e rotas bioquímicas, que são mais ou menos atuantes de acordo com a quantidade do substrato orgânico e das condições químicas e ou bioquímicas do meio onde se processam essas reações (STEVENSON, 1982).

Na rota da polimerização os resíduos são decompostos em substâncias mais simples, chamadas fração leve, que começam a se aglomerar e se polimerizar formando substâncias mais complexas, onde primeiro há formação de ácidos fúlvicos com baixo grau de polimerização, seguida da de ácidos húmicos com grau intermediário e finalmente a humina que é a fração de substancia húmica mais polimerizada e aromática. Além de outros indicadores, a maturação do fertilizante orgânico está diretamente relacionada com a proporção de substâncias húmicas (frações: ácidos fúlvicos, ácidos húmicos e humina), e a quantificação destas frações é um indicador do grau de maturação do composto, e consequentemente, da sua qualidade. As substâncias húmicas informam sobre os processos que regulam ou determinam os benefícios que o fertilizante promoverá no solo e nas plantas (DIAS, 2005).

Queiroz et al. (2014) avaliaram a influência do esterco de coelho, combinado ou não com fertilizante sintético no cultivo do milheto, e concluíram que o esterco de coelho apresentou potencial para mineralização em curto prazo, além de relação $\mathrm{C} / \mathrm{N}$ próxima do valor indicado como referência $(\mathrm{C} / \mathrm{N} \leq 20)$, podendo ser classificado como um fertilizante orgânico simples de acordo com o decreto 4954/2004 (BRASIL, 2004) e em conformidade

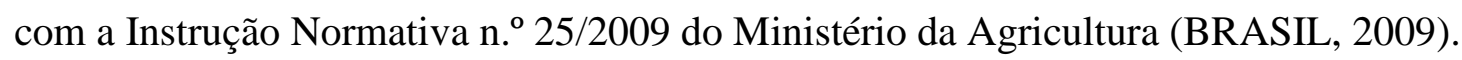

\section{CONCLUSÃO}

O esterco de coelho pode ser estabilizado por meio da ação de larvas de besouros coprófagos da subfamília Cetoniinae que colonizam espontaneamente esse material, produzindo fertilizante orgânico semelhante aos obtidos mediante a sua estabilização por meio de vermicompotagem ou de compostagem, com a vantagem de apresentar menor emissão de $\mathrm{NH}_{3}$ durante o seu processamento.

\section{REFERÊNCIAS BIBLIOGRÁFICAS}

ANJOS, J. L.; AQUINO, A. M.; SCHIEDECK, G. Minhocultura e vermicompostagem: Interface com sistemas de produção, meio ambiente e agricultura de base familiar. Brasília: Embrapa, 2015. 231 p.

ANTUNES, L. F. S. Produção de gongocompostos e sua utilização como substrato para mudas de alface. 2017. 73 f. Dissertação (Mestrado em Agronomia - Ciência do Solo) Universidade Federal Rural do Rio de Janeiro, 2017.

ARAÚJO NETO, S. E.; AZEVEDO, J. M. A.; GALVÃO, R. O.; OLIVEIRA, E. B. L.; FERREIRA, R. L. F. Produção de muda orgânica de pimentão com diferentes substratos. Ciência Rural, Santa Maria, v. 39, n. 5, p.1408-1413, 2009. 
BERNAL, M. P.; ALBURQUERQUE, J. A.; MORAL, R. Composting of animal manures and chemical criteria for compost maturity assessment: a review. Bioresource Technology, Amsterdam, v. 100, p.5444-5453, 2009.

BIDONE, F. A. (org.). Resíduos sólidos provenientes de coletas especiais: eliminação e valorização. Brasília: FINEP/PROSAB, 2001. 216 p.

BRASIL. Lei Federal $n^{\circ} 10.831$, de 23 de dezembro de 2003. Dispõe sobre a agricultura orgânica, e dá outras providências. Diário Oficial da República Federativa do Brasil, Brasília, DF, Seção 1, p. 8, 24 dez. 2003. Disponível em: http://www.planalto.gov.br/ccivil_03/leis/2003/L10.831.htm. Acesso em: 04 out. 2017.

BRASIL. Ministério da Agricultura, Pecuária e Abastecimento. Decreto n. ${ }^{\circ} 4.954$ de 14 de jan. 2004. Diário Oficial da União. Brasília, Seção 1, p. 2, 15 jan. 2004.

BRASIL. Ministério da Agricultura, Pecuária e Abastecimento. Secretaria de Defesa Agropecuária. Instrução Normativa n. 25, de 23 de julho de 2009. Normas sobre as especificações e as garantias, as tolerâncias, o registro, a embalagem e a rotulagem dos fertilizantes orgânicos simples, mistos, compostos, organominerais e biofertilizantes destinados à agricultura. Diário Oficial da República Federativa do Brasil, Brasília, Seção 1, 28 jul. 2009.

BRASIL. Ministério da Agricultura, Pecuária e Abastecimento. Instrução Normativa SDA N. ${ }^{\circ}$ 31. Alteração dos subitens 3.1.2, 4.1 e 4.1.2 da Instrução Normativa n. ${ }^{\circ} 17$ de 21/05/2007. Métodos Analíticos Oficiais para Análise de Substratos para Plantas e Condicionadores de Solo. Diário Oficial da União, Brasília, Seção 1, 24 out. 2008.

BRITO, L. M. C. M. Manual de compostagem. Refóios do Lima: Escola Superior Agrária de Ponte de Lima, Instituto Superior de Viana do Castelo, 2005. Disponível em: http://www.ci.esapl.pt/mbrito/compostagem. Acesso em: nov 2016.

CABANILLAS, C.; TABLADA, M.; LEDESMA, A. Vermicompost: Alternative to urea in basil seed production. Management of Environmental Quality: an International Journal, Bingley, v. 24, n. 2, p.165-177, 2013.

CROSSLEY JÚNIOR., B.; MUELLER, R.; PERDUE, J. C. Biodiversity of microarthropods in agricultural soils: relations to process. In: PAOLETTI, M. G.; PIMENTEL, D. (ed.). Biotic diversity in agroecosystems Amsterdam: Elsevier, 1992. cap. 1-4, p. 37-46.

DIAS, B. O. Estoque de carbono e quantificação de substâncias húmicas de latossolo sob aplicação continuada de lodo de esgoto. Caracterização da matéria orgânica de latossolo sob aplicação continuada de lodo de esgoto. 2005. 110 f. Dissertação (Mestrado em solos e Nutrição de Plantas) - Universidade Federal de Lavras, Lavras, 2005.

FERREIRA, D. F. Sisvar: a guide for its Bootstrap procedures in multiple comparisons. Ciência e Agrotecnologia, Lavras, v. 38, n. 2, p.109-112, 2014.

GÓMEZ-BRANDÓN, M. L.; LORES, M, DOMÍNGUEZ, J. Changes in chemical and microbiological properties of rabbit manure in a continuous-feeding vermicomposting system. Bioresource Technology, Amsterdam, v. 128, n. 1, p.310-316, 2013. 
GRAZIANO, T. T.; DEMATÊ, J.B. I; VOLPE, C. A.; PERECIN, D. Interação entre substratos e fertirrigacão na germinação e na produção de mudas Tagetes patula L. (compositae). Revista Brasileira de Horticultura Ornamental, Campinas, v. 1, n. 2, p.78$85,1995$.

HAFNER S. D.; MONTES, F.; ROTZ, C. A. The role of carbon dioxide in emission of ammonia from manure. Atmospheric Environment, Kidlington, v. 66, n. 2, p.63-71, 2013.

HAIMI, J., HUHTA, Y. Capacity of various organic residues to support adequate earthworm biomass for vermicomposting. Biology and Fertility of Soils, Heidelberg, v. 2., n. 1, p.23-21, 1986.

HANDRECK, K., BLACK, N. Growing media for ornamental plants and turf. Sydney: University of New South Wales Press Ltd., 2010. 551 p.

KIEHL, E. J. Fertilizantes orgânicos. São Paulo: Agronômica Ceres, 1985. 492 p.

KIEHL, E. J. Manual de compostagem: maturação e qualidade do composto. Piracicaba: Editado pelo autor 1998. $171 \mathrm{p}$.

KONONOVA, M. M.; DE MUNTAN, E. B. Materia orgánica del suelo: su naturaleza, propiedades y métodos de investigación. Barcelona: Oikos-tau, 1982. 368 p.

LIAO, C. F. H. Devarda's Alloy Method for Total Nitrogen Determination. Soil Science Society of America Journal, Madison, v. 45, n. 5, p.852-855,1981.

LUIZZI, D.; FERREIRA, J. D; SCHNEIDER, M. B. O comércio internacional de produtos orgânicos: atuação do Brasil e de países atuantes no setor. Caderno de Administração, São Paulo, v. 24, n. 2, p.1-17, 2016.

MOLINA, M. J.; SORIANO, M. D.; INGELMO, F.; LLINARES, J. Stabilisation of sewage sludge and vinasse bio-wastes by vermicomposting with rabbit manure using Eisenia fétida. Management of Environmental Quality. Bioresource Technology, Amsterdam, v. 137, n. 6, p.188-197, 2013.

MORSELLI, T. B. G. A.; CRUZ, L. E. C.; POCM, D.; PICH, A. H. Efeito de diferentes resíduos no comportamento de Eisenia foetida em estação quente: ec1osão. In: CONGRESSSO GAÚCHO DE MINHOCULTURA, 1., 1996, Pelotas. Resumos [...] Pelotas: UFPel, 1996. p. 45-49.

OLIVEIRA, E. A. G.; LEAL, M. A. A.; ROCHA, M. S.; GUERRA, J. G. M.; RIBEIRO, R. L. D. Avaliação da estabilidade de materiais orgânicos por meio de incubação e da captura conjunta das emissões de $\mathbf{C O}_{2}$ e de $\mathbf{N H}_{3}$. Seropédica: Embrapa Agrobiologia, 2014. 16 p. (Embrapa Agrobiologia. Boletim de Pesquisa e Desenvolvimento, 97).

PEREIRA, C. M. S. Efeito das larvas de besouro no processo de decomposição de esterco de coelho e produção de substratos orgânicos para mudas de hortaliças. 2017. 65 f. Tese (Doutorado em Ciência, Tecnologia e Inovação Agropecuária) - Universidade Federal Rural do Rio de Janeiro - UFRRJ, Seropédica, 2017.

PUKER, A; AD'VINCULA, H.J; KORASAKI, V.; FERREIRA, F. N. F; OROZCO, J. Biodiversity of Cetoniinae beetles (Coleoptera: Scarabaeidae) in introduced and native habitats in the Brazilian Atlantic Forest. Entomological Science, Richmond, v. 17, n. 3, p.309-315, 2014. 
QUEIROZ, C. R. A. A.; ANDRADE, R. R.; LACERDA, Z. C.; FERREIRA, M. E. Esterco de coelho: fonte de nutrientes para complementação da adubação. Revista Agrogeoambiental, Pouso Alegre, v. 6, n. 3, p.11-17, 2014.

RAVIV, M.; MEDINA, S.; KRASNOVSKY, A.; ZIADNA, H. Organic matter and nitrogen conservation in manure compost for organic agriculture. Compost Science \& Utilization, Philadelphia, v. 12, n. 1, p.6-10, 2004.

RAMANATHAN, B.; ALAGESAN, P. Evaluation of millicompost versus vermicompost. Current Science, Bengaluru, v. 103, n. 2, p.140-143, 2012.

SILVA, F. A. M.; LOPEZ, F. G.; BOAS, R. L. V.; DA SILVA, R. B. Transformação da matéria orgânica em substâncias húmicas durante a compostagem de resíduos vegetais. Revista Brasileira de Agroecologia, Porto Alegre, v. 4, n. 1, p.59-66, 2009.

SOMMER, S. G.; MOLLER, H. B. Emission of greenhouse gases during composting of deep litter from pig production- effect of straw content. Journal of Agricultural Science., Cambridge, v. 134, n.3, p.327-335, 2000.

STEVENSON, F.J., Húmus Chemistry: genesis, composition and reaction. New York: John Wiley; 1982. $443 \mathrm{p}$.

TEST METHODS FOR THE EXAMINATION OF COMPOSTING AND COMPOST TMECC. Field sampling of compost materials: organic and biological properties: respirometry. Holbrook: Composting Council Research \& Education Foundation, 2002. Disponível em: https://www.compostingcouncil.org/page/tmecc. Acesso em: 25 out. 2019.

VALLINI, G. Il Compostaggio. In: BERTINI, I.; CIPOLLINI, R.; TUNDO, P. (Eds.) La protezione dell'ambiente in Italia. Bologna: Consiglio Nazionale delle Richerche, Società Chimica Italiana e Consorzio Interuniversitario Chimica perl'Ambiente, 1995. cap.4 , p. 83134.

WICHUK, K. M.; McCARTNEY, D. Compost stability and maturity evaluation: a literature review. Journal of Environmental Engineering and Science, London, v. 8, n. 5, p.601-620, 2013. 